\title{
Autophagy-Related Genes in Atherosclerosis
}

\author{
Yuankun Chen $\mathbb{D}^{1,2}$ Ao Zeng $\mathbb{D}^{1},{ }^{1}$ Shumiao He $\mathbb{D}^{1,3}$ Siqing He $\mathbb{D}^{1},{ }^{1}$ Chunmei Li $\mathbb{D},{ }^{1,2}$ \\ Wenjie Mei $\mathbb{I D}^{3}$ and Qun Lu $(\mathbb{D})^{1,2,3}$ \\ ${ }^{1}$ School of Life Sciences and Biopharmaceutics, Guangdong Pharmaceutical University, Guangzhou, China \\ ${ }^{2}$ Guangdong Province Key Laboratory of Pharmaceutical Bioactive Substances, Guangdong Pharmaceutical University, \\ Guangzhou, China \\ ${ }^{3}$ Guangdong Province Engineering and Technology Center for Molecular Probe and Bio-medicine Imaging, Guangzhou, China
}

Correspondence should be addressed to Qun Lu; luqun@gdpu.edu.cn

Received 7 May 2021; Revised 2 June 2021; Accepted 22 June 2021; Published 2 July 2021

Academic Editor: Hassène Gritli

Copyright $\odot 2021$ Yuankun Chen et al. This is an open access article distributed under the Creative Commons Attribution License, which permits unrestricted use, distribution, and reproduction in any medium, provided the original work is properly cited.

\begin{abstract}
Background. Atherosclerosis (AS) is a common chronic vascular inflammatory disease and one of the main causes of cardiovascular/cerebrovascular diseases (CVDs). Autophagy-related genes (ARGs) play a crucial part in pathophysiological processes of AS. However, the expression profile of ARGs has rarely been adopted to explore the relationship between autophagy and AS. Therefore, using the expression profile of ARGs to explore the relationship between autophagy and AS may provide new insights for the treatment of CVDs. Methods. The differentially expressed ARGs of the GSE57691 dataset were obtained from the Human Autophagy Database (HADb) and the Gene Expression Omnibus (GEO) database, and the GSE57691 dataset contains 9 aortic atheroma tissues and 10 normal aortic tissues. The differentially expressed ARGs of the GSE57691 dataset were analyzed by protein-protein interaction (PPI), gene ontology analysis (GO), and Kyoto Encyclopedia of Genes and Genomes analysis (KEGG) and were chosen to explore related miRNAs/transcriptional factors. Results. The GSE57691 dataset had a total of 41 differentially expressed ARGs. The GO analysis results revealed that ARGs were mainly enriched in autophagy, autophagosome, and protein serine/threonine kinase activity. KEGG analysis results showed that ARGs were mainly enriched in autophagy-animal and longevity regulating signaling pathways. Expressions of ATG5, MAP1LC3B, MAPK3, MAPK8, and RB1CC1 were regarded as focus in the PPI regulatory networks. Furthermore, 11 related miRNAs and 6 related transcription factors were obtained by miRNAs/transcription factor target network analysis. Conclusions. Autophagy and ARGs may play a vital role in regulating the pathophysiology of AS.
\end{abstract}

\section{Introduction}

Autophagy, a capacity of maintaining cellular homeostasis, is a process of damaging cytosolic material and delivering to lysosomes for degradation, leading to the turnover of cell material and providing macromolecular precursors $[1,2]$. Autophagy deficiency is closely related to multiple diseases, such as cancers, CVDs, and immune disorders $[3,4]$.

CVDs still remain a prevalent cause of mortality and morbidity worldwide, affecting 16.7 millions of individuals each year [5]. One of main causes of acute cardiovascular death is AS. AS is a common chronic inflammatory disease characterized by atherosclerotic plaque and vascular stenosis $[6,7]$.
Studies have shown that autophagy may be involved in regulating cell survival and death during the occurrence and development of AS $[8,9]$. In the early stage of atherosclerotic lesions, autophagy can inhibit the apoptosis of vascular endothelial cells and delay the development of atherosclerotic plaques. In the late stage of atherosclerotic lesions, excessive activation of autophagy leads to autophagic death of vascular cells, decreased collagen synthesis, and weak fibrous caps that cause plaque rupture. Vascular smooth muscle cells (VSMCs) play a significant role in atherogenesis. Autophagy in VSMCs protects cells in AS plaque by suppressing oxidative stress, inhibiting mitochondrial depolarization and degrading damaged substances [10]. Furthermore, successful autophagy inhibits VSMCs senescence, 
whereas defective autophagy accelerates atherogenesis. The role of autophagy in AS remains undefined though a series of studies have reported that autophagy is activated in AS.

In the past few years, bioinformatics and microarray technologies have been widely used to excavate the genetic targets of multiple diseases, which have helped researchers in identifying the differentially expressed genes and potentially different signaling pathways. However, few research studies applied to explore the relationship between autophagy and AS.

In this study, we used ARGs to explore the relationship between AS and autophagy. The differentially expressed ARGs were screened, and the relevant data were processed by GO and KEGG analysis. The complex interaction between miRNAs/transcriptional factors and genes was predicted. This main aim of study is to explore the relationship between autophagy and AS.

\section{Methods}

2.1. Data Collection. A total of 232 ARGs were gathered from the HADb (http://www.autophagy.lu/index.html) [11]. GEO (http://www.ncbi.nlm.nih.gov/geo) is a highthroughput resource functional genomics database that includes chips, microarrays, and gene expression data [12]. GSE57691 dataset was obtained from the GEO database, which contains 9 aortic atheroma tissues and 10 normal aortic tissues. Meanwhile, the probe is converted into a homologous gene symbol using the annotation information on the platform.

2.2. Differentially Expressed ARGs. The limma method in $R$ was used to analyze the differential expression of ARGs between the AS group and control group [13]. The threshold was the adjusted $P$ value $<0.05$ and $\mid \log 2$ fold change (FC) | $>2$ [14]

2.3. Data Repeatability Test. The intragroup data repeatability of each group was verified by Pearson's correlation. $R$ programming language provides operating environment and software for drawing of graphs and statistical analysis [15]. Use heatmaps to visualize correlations between samples in the same dataset and using $R$ to draw the heatmap. Principal component analysis (PCA) is a sample clustering method for gene expression, diversity analysis, and resequencing [16]. The sample cluster analysis method is used to verify the intragroup data repeatability of the dataset.

2.4. GO and KEGG Online Enrichment Analysis. The enrichment analysis of GO [17] and KEGG [18] in ARGs were performed through DAVID (version 6.8, Database for Annotation, Visualization, and Integrated Discovery, https://david.ncifcrf.gov/) [19]. The visual GO and KEGG enrichment plots of annotation results were analyzed through "digest" and "GO plot" packages in $R$ [20].

2.5. PPI Regulatory Network Construction. Evaluate the interaction between ARGs through the STRING database (version 11.0, https://string-db.org/) [21]. In addition, a combined score $>0.4$ was considered to be a statistically significant interaction. Then, the PPI regulatory network analysis results are loaded into Cytoscape for visualization $[22,23]$.

2.6. miRNAs/Transcription Factor Networks Construction. The miRNAs/transcription factor was analyzed by WebGestalt (http://www.webgestalt.org/) [24]. The correlation between the miRNA/transcription factor and ARGs in significant clustered modules was analyzed by overrepresentation enrichment analysis (http://amp.pharm.mssm. edu/Enrichr/) [25]. Finally, Cytoscape was used to visualize the miRNAs/transcription factor network [24].

\section{Results}

3.1. Repeatability Verification of Dataset. We used the PCA and Pearson's correlation test to validate the GSE57691 dataset repeatability. According to the PCA, the intragroup repeatability of the GSE57691 dataset was acceptable. In the control group and the AS group, the distances between per sample were close in the dimension of principal component 1 . In addition, the distances between the control group and the AS group were far (Figure 1(b)). Pearson's correlation test revealed that there was a strong correlation among the samples in the control group and there was a strong correlation among the samples in the AS group of the GSE57691 dataset. Moreover, sample in the control group and sample in the AS group exist a negative correlation (Figure 1(a)).

\subsection{Differentially Expressed ARGs Identified between the AS} Group and Control Group. In the GSE57691 dataset, 41 differentially expressed ARGs, including 20 upregulated ARGs (TBK1, BID, GNAI3, PTK6, and so on) and 21 downregulated ARGs (ITGA3, WIPI2, MAPK3, FADD, and so on), were identified in the AS group, as shown in heatmap (Figure 2(a)). In addition, the differentially expressed ARGs between the control group and AS group are shown in volcano plot (Figure 2(b)).

\subsection{Functional and Pathway of Differentially Expressed ARGs} Enrichment Analysis. Based on the GO analysis, we found that the biological processes of differentially expressed ARGs were markedly enriched in autophagy, process utilizing autophagic mechanism, and macroautophagy. The cell components of differentially expressed ARGs were markedly enriched in autophagosome, vacuolar membrane, and phagophore assembly site membrane. The molecular function of differentially expressed ARGs was markedly enriched in protein serine/ threonine kinase activity, MAP kinase activity, and ubiquitin protein ligase binding (Figure 3(a)). The results of the KEGG analysis showed that differentially expressed ARGs were primarily enriched in the autophagy-animal signaling pathway and longevity regulating signaling pathway (Figure 3(b)).

3.4. PPI Regulatory Network and Module Analysis, Hub Gene Identification and Analysis. The PPI regulatory network of differentially expressed ARGs was constructed (Figure 4(a)). 


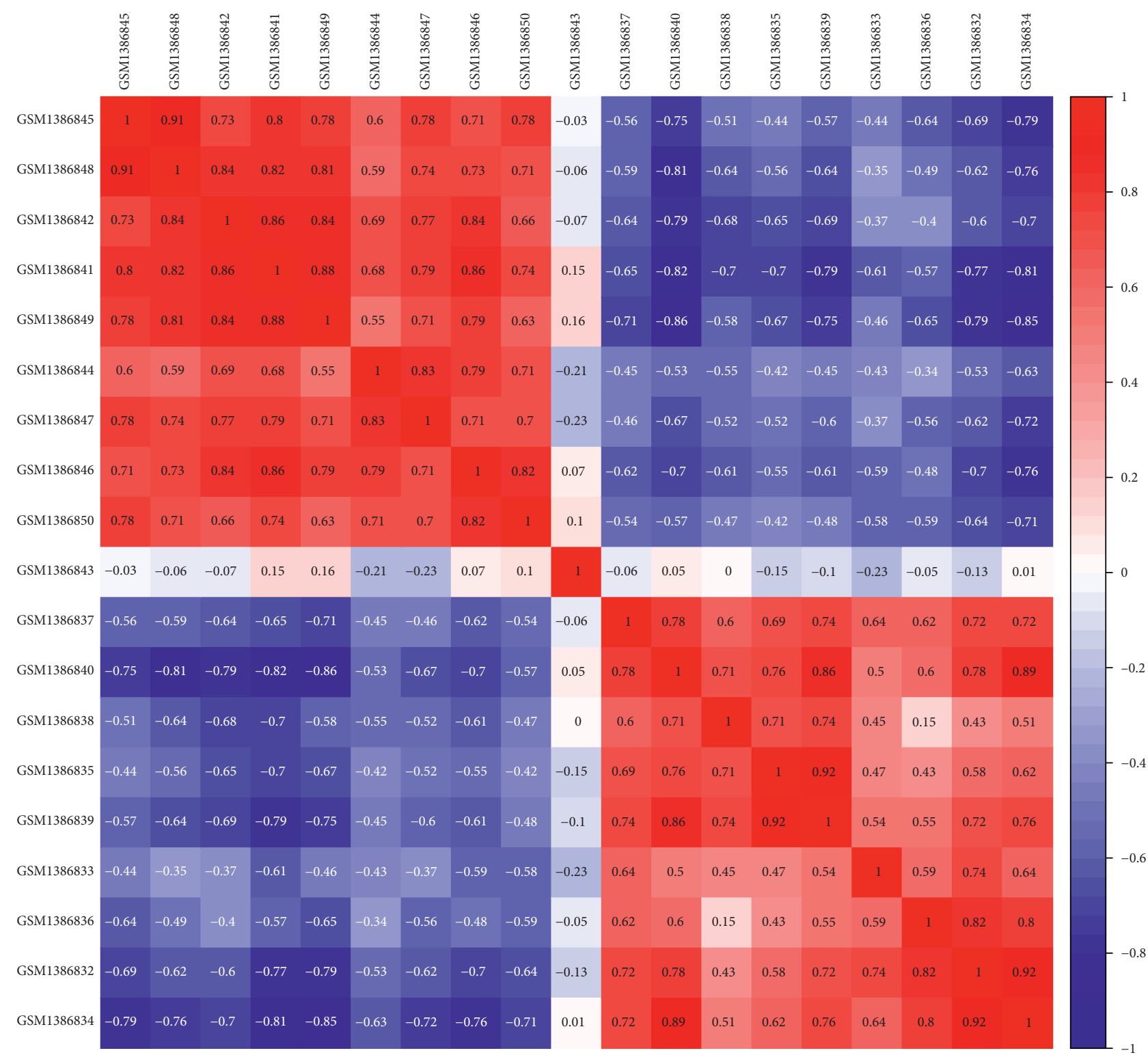

(a)

Figure 1: Continued. 


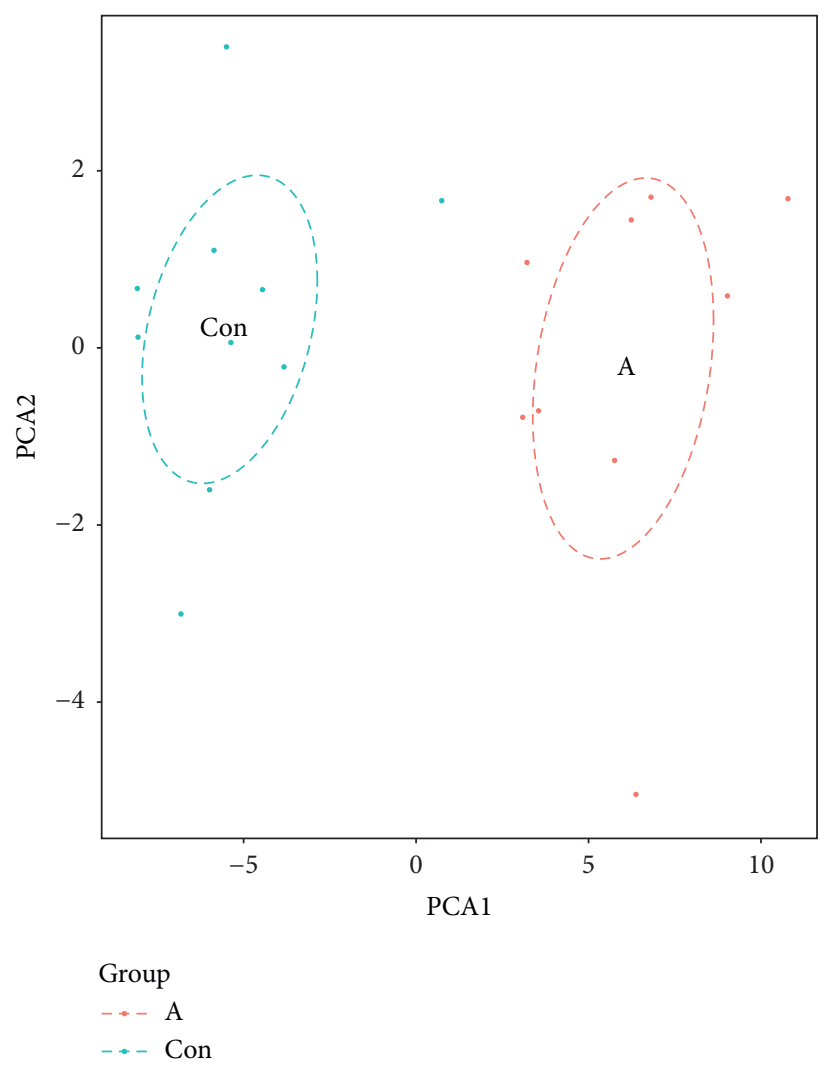

(b)

FIGURE 1: Intragroup data repeatability of the GSE57691 dataset verified by Pearson's correlation and PCA analysis. (a) Pearson's correlation analysis of intragroup data from the GSE57691 dataset. The color represents the degree of correlation. $0<$ correlation $<1$ indicates a positive correlation, and $-1<$ correlation $<0$ indicates a negative correlation. When the absolute value of a number is large, there exists a strong correlation. (b) PCA analysis of intragroup data from the GSE57691 dataset. In the scatter diagram, PC1 and PC2 represent $X$-axis and $Y$ axis, respectively, where each point is a sample. The distance between the two samples represent the difference in gene expression patterns.

The PPI regulatory networks contain 35 nodes and 135 interacting pairs. Then, the most significant module and hub genes in the network were screened by Cytoscape (Figures $4(\mathrm{~b})$ and $4(\mathrm{c})$ ). A total of 10 hub genes with degrees $\geq 13$ were screened: PTEN, FOXO3, RPS6KB1, MAP1LC3A, ULK1, RB1CC1, MAPK8, MAPK3, MAP1LC3B, and ATG5. In addition, subnet modules $A$ and $B$ were selected in the PPI regulatory network. Module A contains 6 upregulated nodes (WDFY3, ATF4, ATG4D, and so on), 10 downregulated nodes (MAP1LC3A, MAPK3, MAPK8, and so on), and 56 interacting pairs. Module $\mathrm{B}$ contains 3 upregulated nodes (HSPAB, RB1CC1, and ATG5), 1 downregulated nodes (MAP1LC3A), and 5 interacting pairs. The genes in the two modules are given in Table 1.

3.5. miRNAs/Transcription Factor Target Networks Analysis. 11 miRNAs (miR-181 family had the most targets) and 6 transcription factors (CREB, ATF, FREAC2, ATF3, CREBP1CJUN, and AP1) were predicted, and 92 regulatory pairs of miRNA and transcription factor networks were constructed (Figure 5). There were 13 upregulated genes and 16 downregulated genes in the miRNAs/transcription factor networks. In the miRNA networks, 13 regulatory interactions were found between upregulated genes and miRNA, and 16 regulatory pairs were identified between downregulated genes and miRNA. In the transcription factor networks, FREAC2 was predicted to target 3 upregulatory genes (CDKN1B, FOXO1, and GRID2) and 5 downregulatory genes (FOXO3, KLHL24, MAPK3, ULK1, and WIPI2). CREB was predicted to target 3 upregulatory genes (ATG5, EEF2, and ST13) and 2 downregulatory genes (MAP1LC3A and RAB24).

\section{Discussion}

Mounting evidence indicates that autophagy participates in maintaining cardiovascular health. Dysfunction of vascular autophagy is related to the initiation of CVDs [26]. Additionally, ARGs affect AS through regulation of VSMCs phenotypic switching, lipid metabolism, and other biological processes [27, 28].

Gene microarray technology is a new method to explore new biomarkers of diseases. Huang et al. screened 98 differentially expressed genes from AS macrophages through DNA microarray analysis and then identified KDELR3, CD55, and DYNC2H1 as key genes through a series of analysis [29]. Another study predicted that miR126 may be a biomarker of AS through gene microarray 


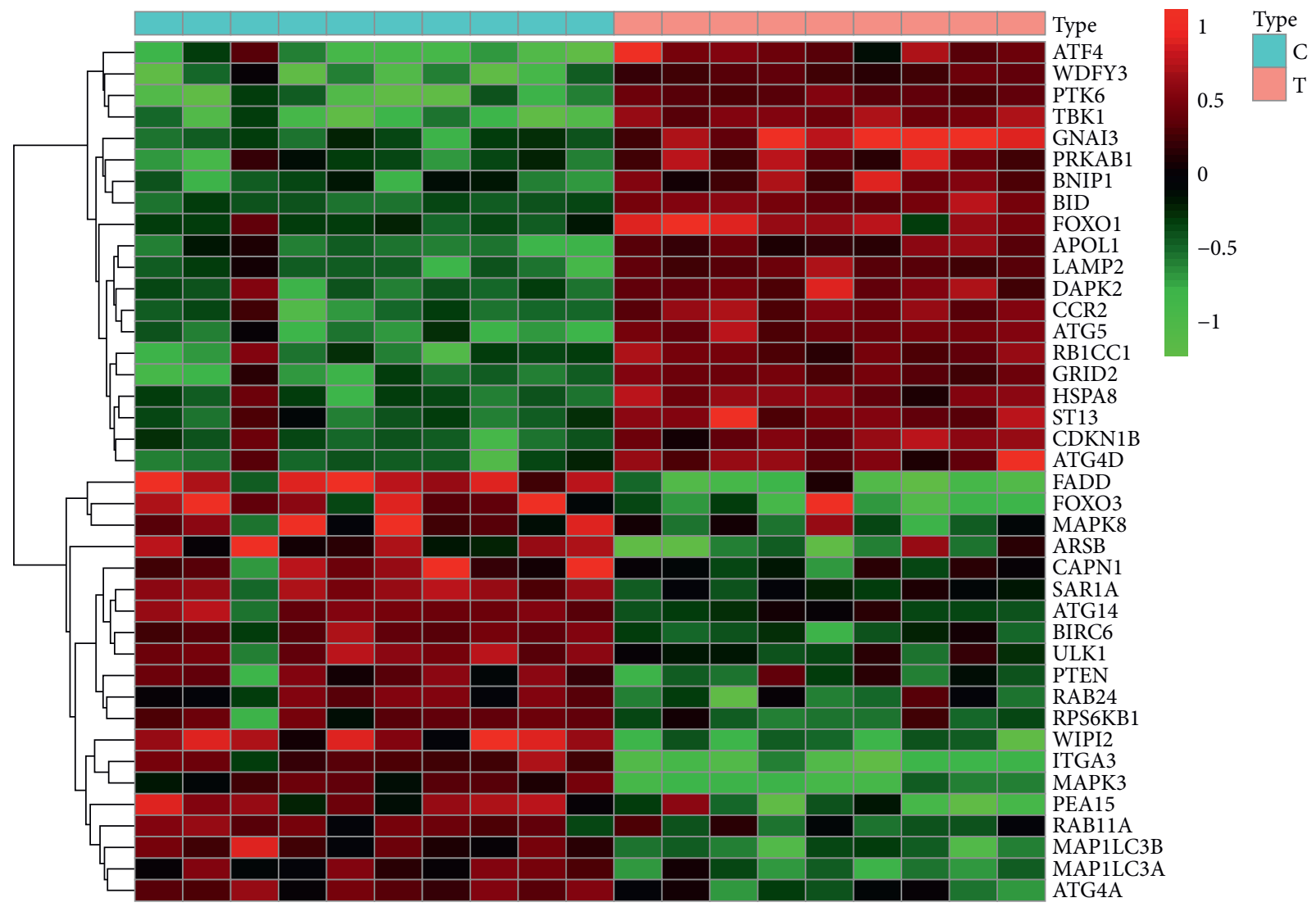

(a)

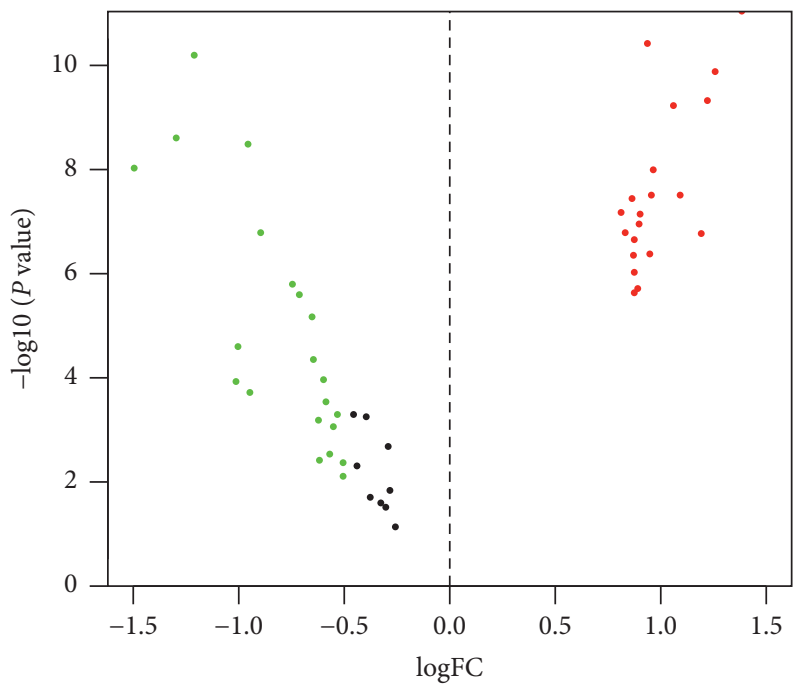

(b)

FIGURE 2: Differential expressions of ARGs between the control group and AS group. (a) The 41 differentially expressed ARGs from the GSE57691 dataset. $C$ indicates the control group and $T$ indicates the AS group. (b) Volcano plot of differentially expressed ARGs. Red indicates high expression genes, green indicates low expression genes, and black indicates that there is no difference in these genes between the AS group and control group.

technology, and its overexpression may prevent the occurrence and development of AS. In addition, lncRNA microarray was also used to study AS. LncRNA-FA2H-2 may improve AS by affecting autophagy and inflammation $[28]$.
In the current study, in order to determine the relationship between ARGs and AS, microarray analysis was used to identify differentially expressed ARGs from the AS group and the control group. First, we screened 41 differentially expressed ARGs between the AS group and control 


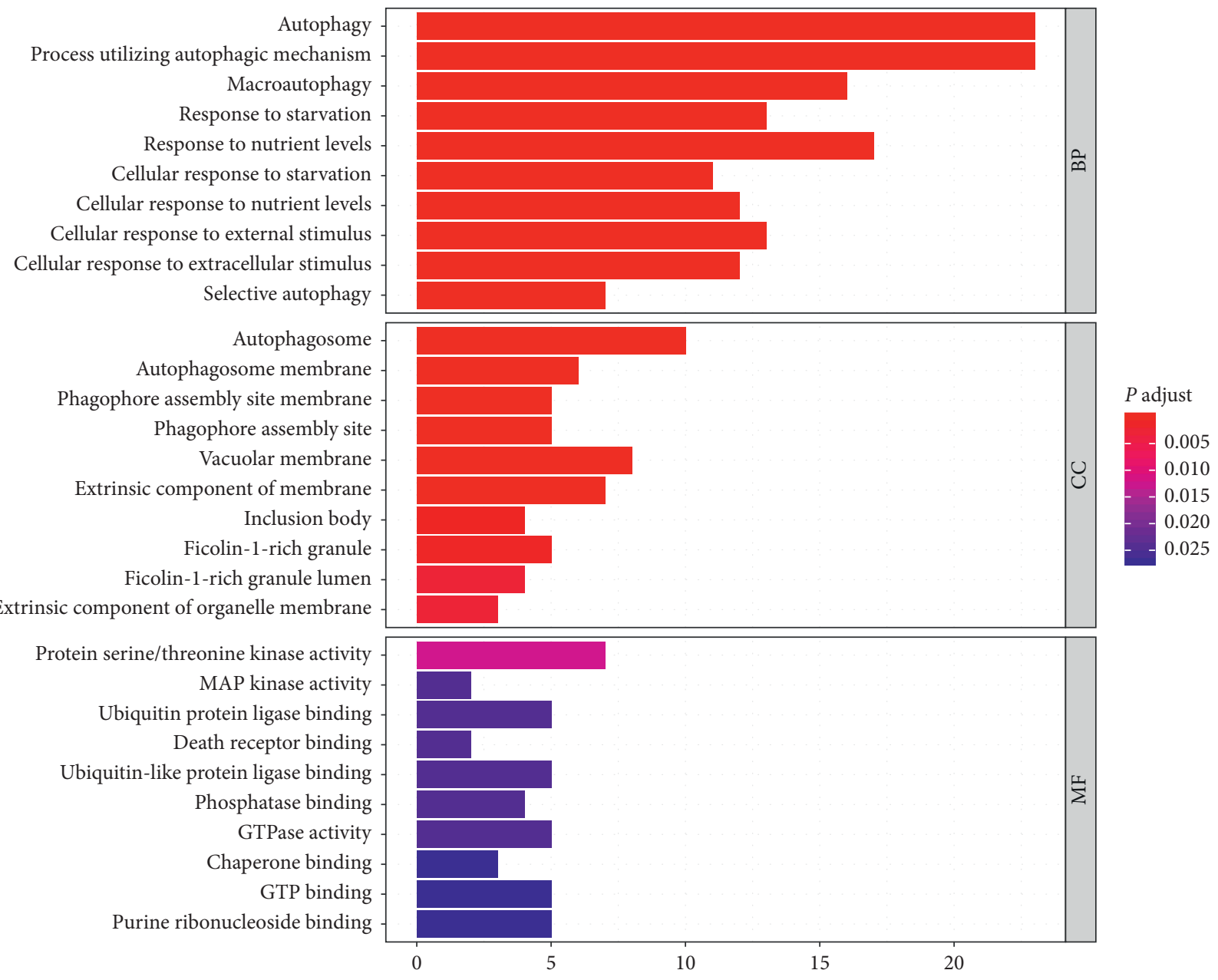

(a)

FIGURE 3: Continued. 


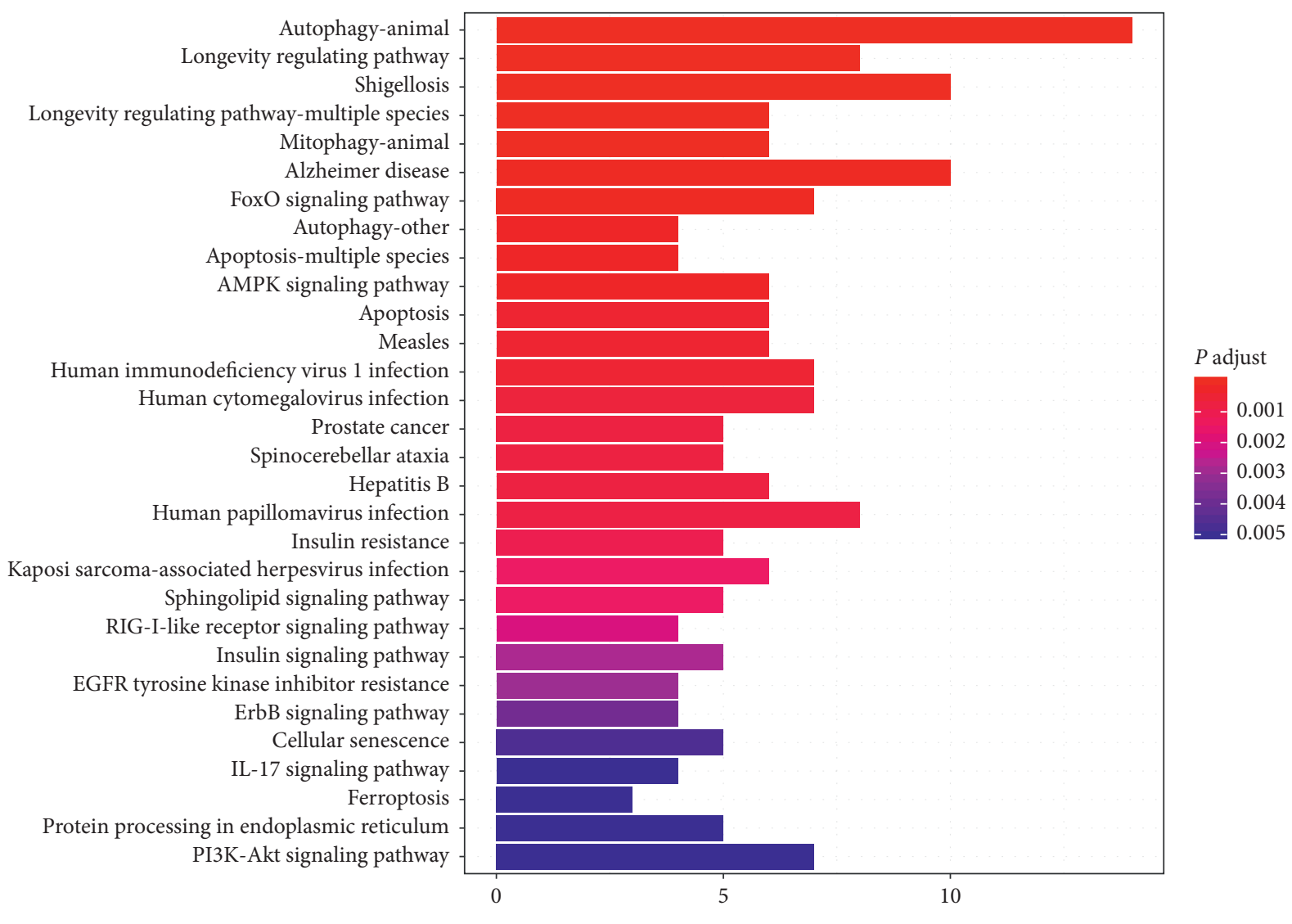

(b)

FIGURE 3: GO and KEGG enrichment analysis of 41 differentially expressed ARGs. (a) Histogram of GO enrichment. (b) Histogram of KEGG enrichment.

group. Based on GO and KEGG analysis, we found that differentially expressed ARGs involve multiple biological processes and signaling pathways, including autophagy, autophagosome, and autophagy-animal signaling pathway. Then, a total of 10 hub genes (PTEN, FOXO3, RPS6KB1, MAP1LC3A, ULK1, RB1CC1, MAPK8, MAPK3, MAP1LC3B, and ATG5) were identified through the PPI regulatory network. Finally, the miRNAs/transcription factor networks were constructed, including $11 \mathrm{miRNAs}$ and 6 transcription factors.

In the BP analysis, most genes were enriched in autophagy. Osonoi et al. found that the degree of autophagy increased in the atherosclerotic smooth muscle cells of human and rabbit by transmission electron microscopy [30]. In the $\mathrm{CC}$ analysis, most genes were enriched in autophagosome. The main role of autophagosomes is to transfer organelles and proteins to lysosomes for degradation. Zhang et al. reported that CAV-1 controls autophagic flux and the formation of autophagosomes by affecting the cellular localization of autophagosomes in lipid rafts, thus influencing the development of AS [31]. In the MF analysis, protein serine/threonine kinase activity enriched the most genes. $\mathrm{AKT}$, the downstream target of $\mathrm{PI} 3 \mathrm{~K}$, is serine/threonine protein kinase. PI3K/AKT is one of the main pathways of autophagy regulation. Previous studies have reported that
Shen-Yuan-Dan capsule treatment reduces foam cell formation by activating autophagy via affecting the PI3K/AKT/ mTORC1 signaling pathway [32]. The KEGG analysis showed that the autophagy-animal signaling pathway might play a role in AS-related autophagy. The AMPK signaling pathway and mTOR signaling pathway are two major pathways in the autophagy-animal pathway. Wu et al. reported that paeonol inhibits the excessive proliferation of VSMCs by inducing AMPK phosphorylation, reducing mTOR phosphorylation, and upregulating autophagy [33].

A total of 10 hub targets were identified in the PPI regulatory network, including ATG5, MAP1LC3B, MAPK3, MAPK8, and RB1CC1. Previous study reported that ATG5 is involved in autophagosomes formation [34]. In addition, ursolic acid exerted antiatherosclerosis effects and protected human umbilical vein endothelial cells (HUVECs) from oxLDL induced cytotoxicity. The underlying mechanism is associated with increased SIRT1 expression, reduced acetylation of lysine residue on Atg5, and enhanced autophagy [35]. These suggest that ATG5 may play an important role in autophagy associated with AS. MAP1LC3B is indirectly related to plaque instability, which may prevent atherosclerotic plaque instability by promoting basic autophagy activity. Meanwhile, Bhairavi Swaminathan et al. found that low expression of MAP1LC3B in carotid atherosclerotic 


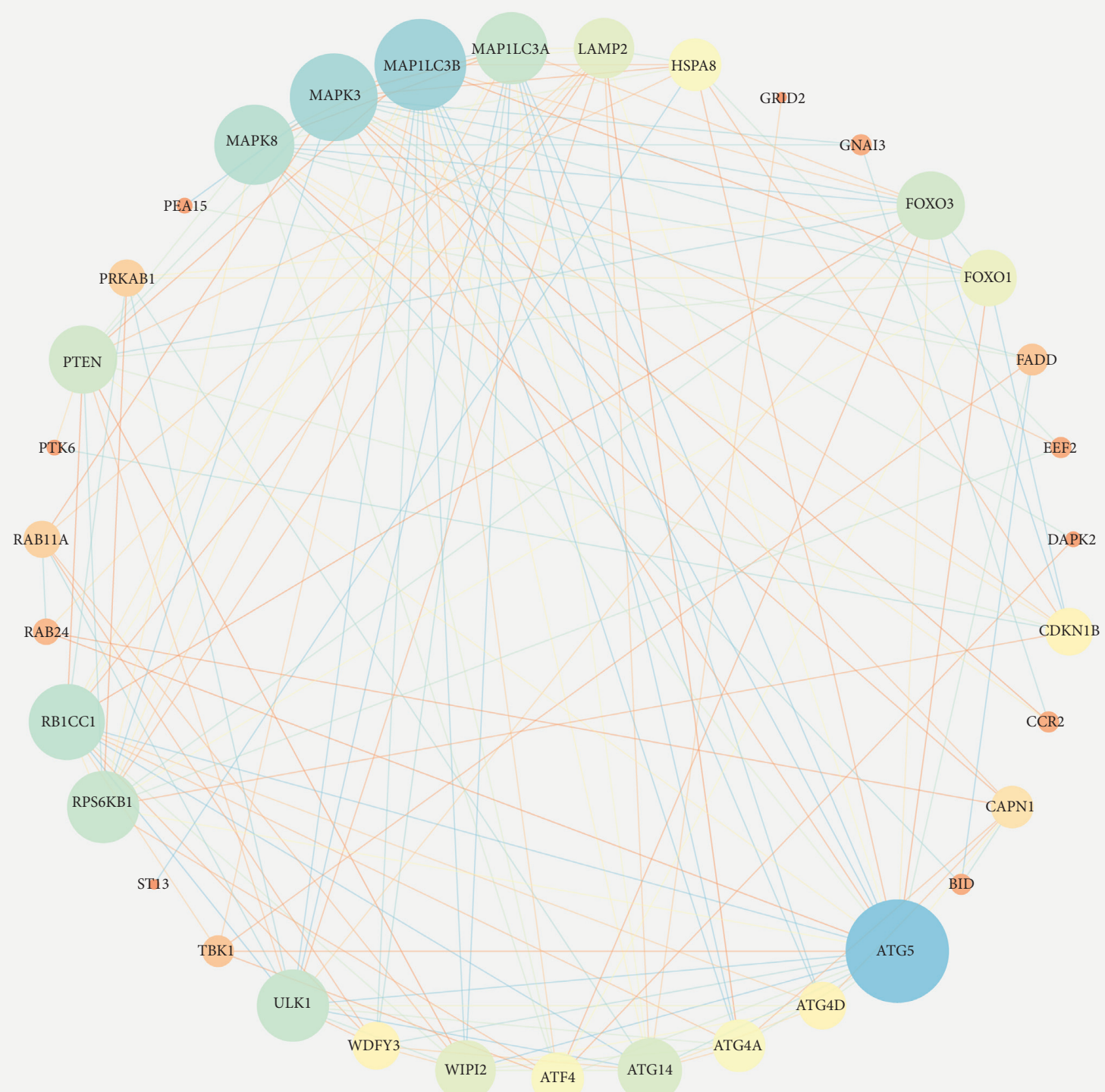

(a)

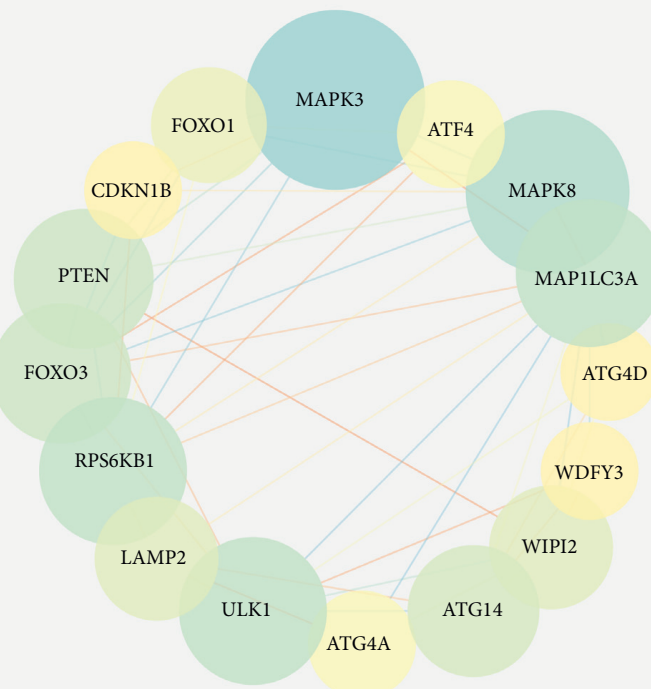

(b)

(c)

FIGURE 4: PPI regulatory network and subnet module analysis of differentially expressed ARGs. The nodes represent the ARGs, and the lines indicate the interaction of two ARGs. The size and color of nodes are positively correlated with the degree and closeness centrality. (a) PPI regulatory networks. (b), (c) Subnet module analysis of PPI regulatory networks. 
TABLE 1: Submodule ARGs and degree of PPI regulatory networks.

\begin{tabular}{|c|c|c|c|c|c|}
\hline \multicolumn{3}{|c|}{ Module A } & \multicolumn{3}{|c|}{ Module B } \\
\hline Node & Description & Degree & Node & Description & Degree \\
\hline WDFY3 & $\mathrm{Up}$ & 8 & HSPA8 & $\mathrm{Up}$ & 9 \\
\hline ATG4D & $\mathrm{Up}$ & 8 & RB1CC1 & $\mathrm{Up}$ & 15 \\
\hline CDKN1B & Up & 8 & MAP1LC3B & Down & 19 \\
\hline ATG4A & Down & 9 & ATG5 & Up & 22 \\
\hline ATF4 & $\mathrm{Up}$ & 9 & & & \\
\hline FOXO1 & $\mathrm{Up}$ & 10 & & & \\
\hline WIPI2 & Down & 11 & & & \\
\hline LAMP2 & $\mathrm{Up}$ & 11 & & & \\
\hline ATG14 & Down & 12 & & & \\
\hline PTEN & Down & 13 & & & \\
\hline FOXO3 & Down & 13 & & & \\
\hline RPS6KB1 & Down & 14 & & & \\
\hline MAP1LC3A & Down & 14 & & & \\
\hline ULK1 & Down & 14 & & & \\
\hline MAPK8 & Down & 16 & & & \\
\hline MAPK3 & Down & 18 & & & \\
\hline
\end{tabular}

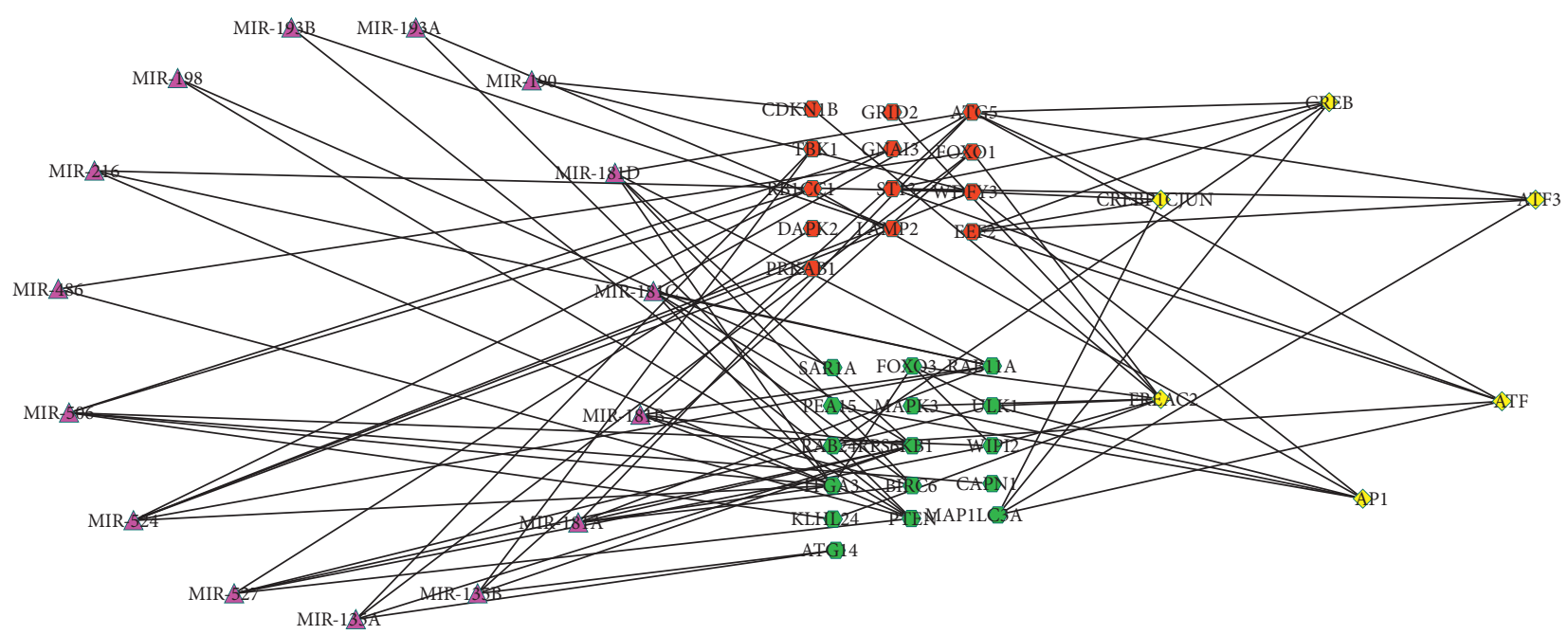

FIGURE 5: miRNAs/transcription factor target networks. The red circles indicate the upregulated ARGs, and the green circles indicate the downregulated ARGs. The violet triangle indicates miRNAs, and the yellow quadrilateral indicates transcription factors.

plaques did not induce related autophagy. This may cause dead cells to accumulate at the site of the lesion and subsequently lead to cerebrovascular events [36]. MAPK3 (ERK1) and MAPK8 (JNK1) are members of the mitogenactivated protein kinase family (MAPK). MAPK signaling pathway plays a vital role in the pathogenesis of CVDs [37]. Babaev et al. found that the reduction of JNK1 in macrophages protects them from apoptosis and increases cell survival rate, thus accelerating early AS [38]. In addition, Zheng et al. reported that protocatechuic acid improved vulnerable lesions in mice, which may be caused by the upregulation of MERTK to normalize arterial inflammation and inhibit MAPK3/1 in lesional macrophages [39]. RB1CC1 is involved in the composition of ULK1-ATG13RB1CC1/RB1CC1-ATG101 complex, which is crucial for the formation of autophagosome [40, 41]. RB1CC1 is also involved in protein synthesis, cell proliferation and migration, differentiation, and cell cycle processes [42]. However, RB1CC1 is rarely reported on CVDs.

Based on the analysis of miRNAs/transcription factor target networks, we found that miR-506 had 7 targeted ARGs, which exerted the most obvious target interaction among the 10 analyzed miRNAs. In particular, miR-506 regulates the hub gene $\mathrm{RB} 1 \mathrm{CC} 1$, and the highly expressed RB1CCI in the network is downregulated by miR-506. $\mathrm{RB} 1 \mathrm{CC} 1$ is essential for the formation of autophagosomes. In addition, the low expression of CAPN1 in the network was upregulated by miR-506. Calpain is involved in inflammation and AS [43]. Yin et al. reported that the downregulation of Calpain-1 and inactivation of Calpain might be closely related to the anti-inflammatory and antiatherosclerosis effects of simvastatin [44]. Therefore, the 
axes of miR-506-CAPN1 and miR-506-RB1CC1 may be closely related to AS and autophagy $[45,46]$.

\section{Conclusion}

In summary, our current study has evaluated the expression of ARGs in AS based on the GEO database and HADb. We found that ARGs were involved in the occurrence and development of AS through multiple biological processes and signaling pathways, such as autophagy, process utilizing autophagic mechanism, macroautophagy, and autophagyanimal signaling pathway. In addition, we also found that the axes of miR-506-CAPN1 and miR-506-RB1CC1 may be closely related to AS and autophagy. Taken all of these, ARGs may play a vital role in AS.

\section{Data Availability}

The data used to support the findings of this study have not been made available because authors were not given permission to share the data.

\section{Disclosure}

No funding bodies had any role in study design, data collection and analysis, decision to publish, or preparation of the manuscript.

\section{Conflicts of Interest}

The authors declare that they have no conflicts of interest.

\section{Authors' Contributions}

Y. K. Chen contributed significantly to analysis and manuscript preparation. A. Zeng, S. M. He, S. Q. He, C. M. Li, and W. J. Mei helped perform the analysis with constructive discussions. Q. Lu helped perform the analysis with constructive discussions.

\section{Acknowledgments}

The authors thank their colleagues from the School of Life Sciences and Biopharmaceutics, Guangdong Pharmaceutical University, for their tremendous efforts during this investigation. This work was supported by grants from the $\mathrm{Na}$ tional Natural Science Foundation of China (81503282) and Science and Technology Planning Project of Guangdong Province (2014A020212309).

\section{References}

[1] A. V. Onorati, M. Dyczynski, R. Ojha, and R. K. Amaravadi, "Targeting autophagy in cancer," Cancer, vol. 124, pp. 33073318, 2018.

[2] K. R. Parzych and D. J. Klionsky, "An overview of autophagy: morphology, mechanism, and regulation," Antioxidants and Redox Signaling, vol. 20, pp. 460-473, 2014.

[3] T. S. Hernandez, G. Fabrias, D. Davila et al., "Dihydroceramide accumulation mediates cytotoxic autophagy of cancer cells via autolysosome destabilization," Autophagy, vol. 12, pp. 2213-2229, 2016.

[4] K. G. Lyamzaev, A. V. Tokarchuk, A. A. Panteleeva, A. Y. Mulkidjanian, V. P. Skulachev, and B. V. Chernyak, "Induction of autophagy by depolarization of mitochondria," Autophagy, vol. 14, pp. 921-924, 2018.

[5] D. C. Goff, A. G. Bertoni, H. Kramer et al., "Dyslipidemia prevalence, treatment, and control in the Multi-Ethnic Study of Atherosclerosis (MESA): gender, ethnicity, and coronary artery calcium," Circulation, vol. 113, pp. 647-656, 2006.

[6] P. C. Evans and B. R. Kwak, "Biomechanical factors in cardiovascular disease," Cardiovascular Research, vol. 99, pp. 229-231, 2013.

[7] G. Sirimarco, P. Amarenco, J. Labreuche et al., "Carotid atherosclerosis and risk of subsequent coronary event in outpatients with atherothrombosis," Stroke, vol. 44, pp. 373-379, 2013.

[8] E. Marzetti, A. Csiszar, D. Dutta, G. Balagopal, R. Calvani, and C. Leeuwenburgh, "Role of mitochondrial dysfunction and altered autophagy in cardiovascular aging and disease: from mechanisms to therapeutics," American Journal of PhysiologyHeart and Circulatory Physiology, vol. 305, pp. H459-H476, 2013.

[9] M. Ouimet, H. Ediriweera, M. S. Afonso et al., "microRNA-33 regulates macrophage autophagy in atherosclerosis," Arteriosclerosis, Thrombosis, and Vascular Biology, vol. 37, pp. 1058-1067, 2017.

[10] D. Gatica, M. Chiong, S. Lavandero, and D. J. Klionsky, "Molecular mechanisms of autophagy in the cardiovascular system," Circulation Research, vol. 116, pp. 456-467, 2015.

[11] N. N. Wang, J. Dong, L. Zhang et al., "HAMdb: a database of human autophagy modulators with specific pathway and disease information," Journal of Cheminformatics, vol. 10, 2018.

[12] T. Barrett, S. E. Wilhite, P. Ledoux et al., "NCBI GEO: archive for functional genomics data sets--update," Nucleic Acids Research, vol. 41, pp. D991-D995, 2013.

[13] I. Diboun, L. Wernisch, C. A. Orengo, and M. Koltzenburg, "Microarray analysis after RNA amplification can detect pronounced differences in gene expression using limma," BMC Genomics, vol. 7, p. 252, 2006.

[14] Y. Guo, Y. Ma, Y. Zhang et al., "Autophagy-related gene microarray and bioinformatics analysis for ischemic stroke detection," Biochemical and Biophysical Research Communications, vol. 489, pp. 48-55, 2017.

[15] X. W. Chen and J. X. Gao, "Big data bioinformatics," Methods, vol. 111, pp. 1-2, 2016.

[16] A. Giuliani, "The application of principal component analysis to drug discovery and biomedical data," Drug Discovery Today, vol. 22, pp. 1069-1076, 2017.

[17] M. Ashburner, C. A. Ball, J. A. Blake et al., "Gene ontology: tool for the unification of biology. The Gene Ontology Consortium," Nature Genetics, vol. 25, pp. 25-29, 2000.

[18] J. Du, Z. Yuan, Z. Ma, J. Song, X. Xie, and Y. Chen, "KEGGPATH: Kyoto encyclopedia of genes and genomes-based pathway analysis using a path analysis model," Molecular BioSystems, vol. 10, pp. 2441-2447, 2014.

[19] D. W. Huang, B. T. Sherman, and R. A. Lempicki, "Systematic and integrative analysis of large gene lists using DAVID bioinformatics resources," Nature Protocols, vol. 4, pp. 44-57, 2009.

[20] W. Walter, C. F. Sanchez, and M. Ricote, "GOplot: an R package for visually combining expression data with functional analysis," Bioinformatics, vol. 31, pp. 2912-2914, 2015. 
[21] D. Szklarczyk, A. Franceschini, M. Kuhn et al., "The STRING database in 2011: functional interaction networks of proteins, globally integrated and scored," Nucleic Acids Research, vol. 39, pp. D561-D568, 2011.

[22] M. Ni, X. Liu, J. Wu et al., "Identification of candidate biomarkers correlated with the pathogenesis and prognosis of non-small cell lung cancer via integrated bioinformatics analysis," Frontiers in Genetics, vol. 9, p. 469, 2018.

[23] P. Shannon, A. Markiel, O. Ozier et al., "Cytoscape: a software environment for integrated models of biomolecular interaction networks," Genome Research, vol. 13, pp. 2498-2504, 2003.

[24] B. Zhang, S. Kirov, and J. Snoddy, "WebGestalt: an integrated system for exploring gene sets in various biological contexts," Nucleic Acids Research, vol. 33, pp. W741-W748, 2005.

[25] A. Maertens, M. Bouhifd, L. Zhao et al., "Metabolomic network analysis of estrogen-stimulated MCF-7 cells: a comparison of overrepresentation analysis, quantitative enrichment analysis and pathway analysis versus metabolite network analysis," Archiv für Toxikologie, vol. 91, pp. 217-230, 2017.

[26] M. D. G. De, M. G. R. De, and W. Martinet, "Autophagy as an emerging therapeutic target for age-related vascular pathologies," Expert Opinion on Therapeutic Targets, vol. 24, pp. 131-145, 2020.

[27] M. O. J. Grootaert, M. Moulis, L. Roth et al., "Vascular smooth muscle cell death, autophagy and senescence in atherosclerosis," Cardiovascular Research, vol. 114, pp. 622-634, 2018.

[28] S. Tai, X. Q. Hu, D. Q. Peng, S. H. Zhou, and X. L. Zheng, "The roles of autophagy in vascular smooth muscle cells," International Journal of Cardiology, vol. 211, pp. 1-6, 2016.

[29] H. M. Huang, X. Jiang, M. L. Hao et al., "Identification of biomarkers in macrophages of atherosclerosis by microarray analysis," Lipids in Health and Disease, vol. 18, p. 107, 2019.

[30] Y. Osonoi, T. Mita, K. Azuma et al., "Defective autophagy in vascular smooth muscle cells enhances cell death and atherosclerosis," Autophagy, vol. 14, pp. 1991-2006, 2018.

[31] X. Zhang, C. M. Ramirez, B. Aryal et al., "Cav-1 (Caveolin-1) deficiency increases autophagy in the endothelium and attenuates vascular inflammation and atherosclerosis," Arteriosclerosis, Thrombosis, and Vascular Biology, vol. 40, pp. 1510-1522, 2020.

[32] M. Zhou, P. Ren, Y. Zhang et al., "Shen-yuan-dan Capsule attenuates atherosclerosis and foam cell formation by enhancing autophagy and inhibiting the PI3K/Akt/mTORC1 signaling pathway," Frontiers in Pharmacology, vol. 10, p. 603, 2019.

[33] H. Wu, A. Song, W. Hu, and M. Dai, "The anti-atherosclerotic effect of paeonol against vascular smooth muscle cell proliferation by up-regulation of autophagy via the AMPK/ mTOR signaling pathway," Frontiers in Pharmacology, vol. 8, p. 948, 2017.

[34] W. Zheng, W. Xie, D. Yin, R. Luo, M. Liu, and F. Guo, “ATG5 and ATG7 induced autophagy interplays with UPR via PERK signaling," Cell Communication and Signaling, vol. 17, p. 42, 2019.

[35] Q. Jiang, R. Hao, W. Wang, H. Gao, and C. Wang, "SIRT1/ Atg5/autophagy are involved in the antiatherosclerosis effects of ursolic acid," Molecular and Cellular Biochemistry, vol. 420, pp. 171-184, 2016.

[36] B. Swaminathan, H. Goikuria, R. Vega et al., "Autophagic marker MAP1LC3B expression levels are associated with carotid atherosclerosis symptomatology," PLoS One, vol. 9, Article ID e115176, 2014.
[37] A. J. Muslin, "MAPK signalling in cardiovascular health and disease: molecular mechanisms and therapeutic targets," Clinical Science, vol. 115, pp. 203-218, 2008.

[38] V. R. Babaev, M. Yeung, E. Erbay et al., "Jnk1 deficiency in hematopoietic cells suppresses macrophage apoptosis and increases atherosclerosis in low-density lipoprotein receptor null mice," Arteriosclerosis, Thrombosis, and Vascular Biology, vol. 36, pp. 1122-1131, 2016.

[39] J. Zheng, Q. Li, L. He et al., "Protocatechuic acid inhibits vulnerable atherosclerotic lesion progression in older apoe-/mice," Journal of Nutrition, vol. 150, pp. 1167-1177, 2020.

[40] N. Gammoh, O. Florey, M. Overholtzer, and X. Jiang, "Interaction between FIP200 and ATG16L1 distinguishes ULK1 complex-dependent and -independent autophagy," Nature Structural \& Molecular Biology, vol. 20, pp. 144-149, 2013.

[41] J. Yao, L. Jia, N. Khan et al., "Deletion of autophagy inducer RB1CC1 results in degeneration of the retinal pigment epithelium," Autophagy, vol. 11, pp. 939-953, 2015.

[42] L. Li, G. Wang, J. S. Hu et al., "RB1CC1-enhanced autophagy facilitates PSCs activation and pancreatic fibrogenesis in chronic pancreatitis," Cell Death \& Disease, vol. 9, p. 952, 2018.

[43] S. Samantaray, V. H. Knaryan, D. C. Shields, A. A. Cox, A. Haque, and N. L. Banik, "Inhibition of Calpain activation protects MPTP-induced nigral and spinal cord neurodegeneration, reduces inflammation, and improves gait dynamics in mice," Molecular Neurobiology, vol. 52, pp. 1054-1066, 2015.

[44] M. Yin, Q. Liu, L. Yu et al., "Downregulations of CD36 and calpain-1, Inflammation, and atherosclerosis by Simvastatin__in apolipoprotein E knockout mice," Journal of Vascular Research, vol. 54, pp. 123-130, 2017.

[45] E. Kyriakakis, A. Frismantiene, B. Dasen et al., "T-cadherin promotes autophagy and survival in vascular smooth muscle cells through MEK1/2/Erk1/2 axis activation," Cellular Signalling, vol. 35, pp. 163-175, 2017.

[46] Z. Song, D. Wei, Y. Chen et al., "Association of astragaloside IV-inhibited autophagy and mineralization in vascular smooth muscle cells with lncRNA H19 and DUSP5-mediated ERK signaling," Toxicology and Applied Pharmacology, vol. 364, pp. 45-54, 2019. 